\title{
Social insects as a model to study the molecular basis of ageing
}

\author{
Laurent Keller*, Stephanie Jemielity \\ Department of Ecology and Evolution, University of Lausanne, Biophore, 1015 Lausanne-Dorigny, Switzerland
}

Received 21 February 2006; received in revised form 3 April 2006; accepted 6 April 2006

\begin{abstract}
One major gap in the current knowledge of the molecular bases of ageing is that most of the work has been done using short-lived model organisms such as fruitflies, nematodes, yeast and mice. Here, we argue that ants and social bee species provide an excellent complementary system to study ageing, and this for two reasons: first, in contrast to model organisms, ant and bee queens are extraordinarily long-lived, and second, there is a tremendous variation in lifespan among the genetically identical queens, workers (non-reproductive females) and males, with queens living up to 500 times longer than males and 10 times longer than workers. We review recent experimental work aimed at testing the role of antioxidant genes within the conceptual framework of the free radical theory of ageing, as well as studies investigating the role of juvenile hormone, vitellogenin and telomeres as mediators of ageing in social insects.
\end{abstract}

(C) 2006 Elsevier Inc. All rights reserved.

Keywords: Ageing; Ant; Genomic; Extreme lifespan

Most candidate genes thought to be involved in ageing have been identified in the model organisms Drosophila melanogaster, Caenorhabditis elegans and the yeast Saccharomyces cerevisiae. All these organisms are extremely short-lived, raising the question of how representative they are of the molecular mechanisms behind ageing in long-lived organisms. Another potential drawback of studying ageing exclusively in model organisms is that all experiments are performed in an artificial environment. Though useful for many obvious reasons, such conditions may in some cases cause results to be misleading. A gene that increases the lifespan of a fruitfly in the laboratory when over-expressed might not have the same effect in a natural population, where an organism faces competition, parasites and other challenges. Thus, although the current candidate genes are able to increase lifespan in a protected environment, some of them might play no relevant role in ageing and lifespan determination under natural conditions. In line with these caveats, several recent studies showed that under stress mimicking natural conditions, some long-lived worm and fruitfly mutants incurred a substantial fitness cost compared to the wildtype (Jenkins et al., 2004; Marden et al., 2003; Walker et al., 2000), suggesting that these mutations could not spread in a natural population.

\footnotetext{
* Corresponding author. Tel.: +412169241 93; fax: +41216924165.

E-mail address: laurent.keller@unil.ch (L. Keller).
}

Because of their peculiar life-history characteristics, social insects are an excellent system to complement the ageing research performed on model organisms. The evolution of sociality in ants, bees, wasps and termites has been accompanied by a 100 -fold increase in the intrinsic (environment-independent) lifespan of the functional females (the queens), most likely because with the advent of social life and division of labour this caste has experienced a spectacular decrease in extrinsic (environment-induced) mortality (Keller and Genoud, 1997). In some ant species, queens can live up to 30 years (Hölldobler and Wilson, 1990). In addition to this extreme lifespan, social insects show a tremendous variation in lifespan among castes within a given species. In ants queens frequently live 10 times as long as workers, despite the fact that both castes generally develop from a single genotype through differential gene expression (Hölldobler and Wilson, 1990; Wheeler, 1986). Similarly, males differ greatly in lifespan from females: in some species queens outlive males by a factor of 500. These characteristics make social insects an excellent testing ground for candidate-ageing and lifespan-regulation genes identified in model organisms. Given that social insect castes are genetically identical, genes and proteins that are truly and globally responsible for differences in lifespan should show different expression and/or activity patterns in ant queens, workers and males, a prediction that is easy to test. Moreover, large-scale gene-expression studies among social insect castes with different lifespans may help identify new candidate genes involved in ageing. 


\section{Studies of the proximate mechanisms of ageing in social insects}

Several recent studies have provided insight into the mechanisms that are likely to mediate lifespan determination in social insects, though this field of research is still in its infancy. These studies tackle ageing-related questions in a variety of ways, but all of them capitalize on the naturally evolved differences in lifespan between castes or between subgroups of individuals within a given caste.

Plasticity in the rate of ageing can be demonstrated in ant and bee societies better than in other taxa. In these organisms, the queen and worker castes whose intrinsic lifespan differs by one order of magnitude develop from a single genotype, and even within castes the rate of ageing seems to be readily modifiable. For instance, hive bees, which are prevented from becoming foragers, can live up to several times as long as forager bees (Amdam and Page, 2005). In addition, ant queens that are mated-be it to fertile or sterile males-are able to live significantly longer than non-mated queens (Schrempf et al., 2005). A prime candidate for mediating these life-history polyphenisms in social insects is juvenile hormone ( $\mathrm{JH})$. JH has been suggested to regulate ageing-rate plasticity, as well as trade-offs between longevity and fecundity in the fruitfly and in other insects (Flatt et al., 2005).

In line with the above hypothesis, the difference in lifespan between hive and forager bees might at least be partially explained by a JH-mediated shutdown of vitellogenin synthesis, which affects the forager immune system (Amdam et al., 2004; Omholt and Amdam, 2004; Seehuus et al., 2006). Switching to foraging is associated with an increase in $\mathrm{JH}$ (Fluri et al., 1982), which, in turn, leads to the cessation of vitellogenin synthesis (Pinto et al., 2000). Amdam et al. (2004) provide correlative and in vitro evidence that low vitellogenin titers are likely to impinge on the number of functional hemocytes, which play an important role in insect immune defense. This vitellogenin-hemocyte association was recently confirmed experimentally: a decrease in vitellogenin expression due to RNA interference resulted in a significantly lower number of functional hemocytes than what was found in workers with normal vitellogenin expression (Seehuus et al., 2006). From an evolutionary perspective, the repression of vitellogenin production and ensuing shutdown of the immune system in foragers has been interpreted as an adaptive colonylevel energy-saving strategy. Because foragers experience a higher risk of external mortality than hive bees, the collective investment in the maintenance of the forager soma may be lower than in that of hive bees (Amdam et al., 2004; Omholt and Amdam, 2004).

In addition to its beneficial effect on worker immune function, vitellogenin might also increase the resistance of honeybee workers to oxidative stress. Seehuus et al. (2006) showed that workers with high vitellogenin titers survive paraquat-induced oxidative stress better than workers with low vitellogenin titers. Likewise, workers with RNA-interferenceinduced low vitellogenin activity died significantly faster from paraquat treatment than sham-injected controls with normal vitellogenin activity.

Two studies, in Lasius niger ants and the honey bee, have focused on the free radical theory of ageing (Harman, 1956). This theory suggests that the accumulation of oxidative damage might be a proximate cause of ageing and that organismal lifespan is at least in part determined by the rate of oxidative damage inflicted on cells and tissues. The rate of oxidative damage depends on the balance between reactive oxygen-species (ROS) production and antioxidant defense (Finkel and Holbrook, 2000). Thus, in theory an increased lifespan can be achieved by lowered ROS production, increased antioxidant defense, or a combination of both. In line with the hypothesis that antioxidant activity might mediate longevity, increased levels of antioxidants were found in several Drosophila lines selected for long lifespan (Arking et al., 2002; Dudas and Arking, 1995, reviewed in Finkel and Holbrook, 2000). In addition, transgenic expression of certain antioxidant genes in Drosophila has been shown to increase lifespan, although lifespan extension frequently depended on the genetic background and other particular conditions (reviewed in Aigaki et al., 2002; Finkel and Holbrook, 2000). However, studies in social insects show that increased antioxidant gene expression or protein activity does not seem to be required for the evolution of a long lifespan. In the ant Lasius niger, $\mathrm{Cu}-\mathrm{Zn}$ superoxide dismutase 1 (CuZnSod) expression and CuZnSOD activity were lower or equal in heads, thoraces and abdomen of adult queens compared to the much shorter-lived adult workers and males (Parker et al., 2004). Similar results were obtained by Corona et al. (2005) in the honey bee. These authors measured the expression levels of eight antioxidant genes in the brain, thorax and abdomen of queens and workers of various ages. The antioxidant genes surveyed included CuZnSod, Mn superoxide dismutase 2 and catalase. Compared to 1-month-old workers, 1-year-old and 1-month-old queens had lower or equal levels of antioxidant gene expression in almost all body parts and tissues analysed. These findings in social insects are consistent with previous cross-species comparisons showing lower levels of antioxidants in longer-lived species (Perez-Campo et al., 1998) and suggest that decreased ROS generation is more likely to contribute to the increased lifespan of queens than an enhanced antioxidant defense (Corona et al., 2005; Parker et al., 2004).

Another study in the ant L. niger focused on telomeres, the DNA-protein complexes that constitute the ends of linear chromosomes. Telomere length regulation is thought to be an important aspect of cell maintenance in eukaryotes, since shortened telomeres can lead to various defects, including impaired cell division (Campisi et al., 2001). Although telomere length is correlated with lifespan in humans (Cawthon et al., 2003) and birds (Haussmann et al., 2005), the role of telomeres in ageing and lifespan determination is still under debate. By means of terminal restriction fragment-length analysis Jemielity, Kimura, Parker, Parker, Aviv and Keller (unpublished) tested whether telomere length in ant somatic tissues correlates with caste-specific lifespan in young adults. The short-lived L. niger males did indeed have significantly 
shorter telomeres than the longer-lived queens and workers. These differences most likely arise during early larval development through faster telomere shortening in males compared to queens and workers. Differences in cell proliferation are unlikely to account for the sex-specific telomere length patterns since males are 6-8 times smaller than queens and are therefore likely to undergo fewer cell divisions than queens. In addition, telomerase is active in somatic tissues of all three castes, suggesting that males do possess the molecular machinery to counteract telomere shortening. Based on their own results and similar correlations between sex-specific telomere length and lifespan in rats (Cherif et al., 2003) and humans (Benetos et al., 2001; Jeanclos et al., 2000; Nawrot et al., 2004), Jemielity et al. argue that sexspecific telomere length patterns may ultimately reflect adaptive differences in the level of somatic maintenance. According to the disposable soma theory, the longer-lived sex is predicted to invest more in somatic maintenance (one aspect of which may be telomere length maintenance) than the shorter-lived sex (Kirkwood, 1981; Kirkwood and Rose, 1991). Queens did not, however, have longer telomeres than the shorter-lived workers, possibly because the development of these two castes is still coupled when telomere length is determined.

In the near future, large-scale microarray analysis should make it possible to learn more about social insect ageing. These molecular tools, which have already been developed for the honey bee (Whitfield et al., 2002) and the fire ant Solenopsis invicta (Wang et al. in preparation), should help identify the genes that make social insect queens so much longer-lived than workers and males. Although this approach is purely comparative, it will allow us to designate candidate genes, which can then be tested with methods such as RNA interference. However, the success of this approach will depend on whether these functional tests can be applied on a routine basis or only to specific subsets of genes.

\section{Acknowledgements}

We would like to thank Prof. Beatrix Grubeck-Loebenstein for inviting us to write this review and one anonymous reviewer and David Jemielity for helpful comments on the manuscript. Our work is funded by the Swiss National Science Foundation, the AETAS Foundation for Research into Ageing (Geneva) and the A.R. and J. Leenards Foundation (Lausanne).

\section{References}

Aigaki, T., Seong, K.H., Matsuo, T., 2002. Longevity determination genes in Drosophila melanogaster. Mech. Ageing Dev. 123, 1531-1541.

Amdam, G.V., Page, R.E., 2005. Intergenerational transfers may have decoupled physiological and chronological age in a eusocial insect. Ageing Res. Rev. 4, 398-408.

Amdam, G.V., Simoes, Z.L.P., Hagen, A., Norberg, K., Schroder, K. Mikkelsen, O., Kirkwood, T.B.L., Omholt, S.W., 2004. Hormonal control of the yolk precursor vitellogenin regulates immune function and longevity in honeybees. Exp. Gerontol. 39, 767-773.
Arking, R., Buck, S., Novoseltev, V.N., Hwangbo, D., Lane, M., 2002. Genomic pasticity, energy allocations, and the extended longevity phenotypes of Drosophila. Ageing Res. Rev. 1, 209-228.

Benetos, A., Okuda, K., Lajemi, M., Kimura, M., Thomas, F., Skurnick, J., Labat, C., Bean, K., Aviv, A., 2001. Telomere length as an indicator of biological aging - the gender effect and relation with pulse pressure and pulse wave velocity. Hypertension 37, 381-385.

Campisi, J., Kim, S.H., Lim, C.S., Rubio, M., 2001. Cellular senescence, cancer and aging: the telomere connection. Exp. Gerontol. 36, 1619-1637.

Cawthon, R.M., Smith, K.R., O'Brien, E., Sivatchenko, A., Kerber, R.A., 2003. Association between telomere length in blood and mortality in people aged 60 years or older. Lancet 361, 393-395.

Cherif, H., Tarry, J.L., Ozanne, S.E., Hales, C.N., 2003. Ageing and telomeres: a study into organ- and gender-specific telomere shortening. Nucleic Acids Res. 31, 1576-1583.

Corona, M., Hughes, K.A., Weaver, D.B., Robinson, G.E., 2005. Gene expression patterns associated with queen honey bee longevity. Mech. Ageing Dev. 126, 1230-1238.

Dudas, S.P., Arking, R., 1995. A coordinate upregulation of antioxidant gene activities is associated with the delayed onset of senescence in a long-lived strain of Drosophila. J. Gerontol. Ser. A - Biol. Sci. Med. Sci. 50, B1117B1127.

Finkel, T., Holbrook, N.J., 2000. Oxidants, oxidative stress and the biology of ageing. Nature 408, 239-247.

Flatt, T., Tu, M.P., Tatar, M., 2005. Hormonal pleiotropy and the juvenile hormone regulation of Drosophila development and life history. Bioessays 27, 999-1010.

Fluri, P., Lüscher, H., Wille, H., Gerig, L., 1982. Changes in weight of the pharyngeal gland and haemolymph titres of juvenile hormone, protein and vitellogenin in worker honeybees. J. Insect Physiol. 28, 61-68.

Harman, D., 1956. Aging - a theory based on free-radical and radiation chemistry. J. Gerontol. 11, 298-300.

Haussmann, M.F., Winkler, D.W., Vleck, C.M., 2005. Longer telomeres associated with higher survival in birds. Biol. Lett. 1, 212-214.

Hölldobler, B., Wilson, E.O., 1990. The Ants. Springer, Berlin.

Jeanclos, E., Schork, N.J., Kyvik, K.O., Kimura, M., Skurnick, J.H., Aviv, A., 2000. Telomere length inversely correlates with pulse pressure and is highly familial. Hypertension 36, 195-200.

Jenkins, N.L., McColl, G., Lithgow, G.J., 2004. Fitness cost of extended lifespan in Caenorhabditis elegans. Proc. R. Soc. Lond. Ser. B-Biol. Sci. 271, 2523-2526.

Keller, L., Genoud, M., 1997. Extraordinary lifespans in ants: a test of evolutionary theories of ageing. Nature 389, 958-960.

Kirkwood, T.B.L., 1981. Repair and its evolution: survival versus reproduction. In: Townsend, C.R., Calow, P. (Eds.), Physiological Ecology: An Evolutionary Approach to Resource Use. Blackwell, Oxford, pp. 165-189.

Kirkwood, T.B.L., Rose, M.R., 1991. Evolution of senescence-late survival sacrificed for reproduction. Philos. Trans. R. Soc. B-Biol. Sci. 332, 15-24.

Marden, J.H., Rogina, B., Montooth, K.L., Helfand, S.L., 2003. Conditional tradeoffs between aging and organismal performance of indy long-lived mutant flies. Proc. Natl Acad. Sci. USA 100, 3369-3373.

Nawrot, T.S., Staessen, J.A., Gardner, J.P., Aviv, A., 2004. Telomere length and possible link to $\mathrm{X}$ chromosome. Lancet $363,507-510$.

Omholt, S.W. Amdam, G.V. 2004. Epigenic regulation of aging in honeybee workers. Sci. Aging Knowledge. Environ. (26), p. pe28.

Parker, J.D., Parker, K.M., Sohal, B.H., Sohal, R.S., Keller, L., 2004. Decreased expression of $\mathrm{Cu}-\mathrm{Zn}$ superoxide dismutase 1 in ants with extreme lifespan. Proc. Natl Acad. Sci. USA 101, 3486-3489.

Perez-Campo, R., López-Torres, M., Cadenas, E., Rojas, C., Barja, G., 1998. The rate of free radical production as a determinant of the rate of aging: evidence from the comparative approach. J. Comp. Physiol. B. 168, 149-158.

Pinto, L.Z., Bitondi, M.M.G., Simões, Z.L.P., 2000. Inhibition of vitellogenin synthesis in Apis mellifera workers by a juvenile hormone analogue, pyriproxyfen. J. Insect Physiol. 46, 153-160.

Schrempf, A., Heinze, J., Cremer, S., 2005. Sexual cooperation: mating increases longevity in ant queens. Curr. Biol. 15, 267-270. 
Seehuus, S.C., Norberg, K., Gimsa, U., Krekling, T., Amdam, G.V., 2006. Reproductive protein protects functionally sterile honey bee workers from oxidative stress. Proc. Natl Acad. Sci. USA 103, 962-967.

Walker, D.W., McColl, G., Jenkins, N.L., Harris, J., Lithgow, G.J., 2000. Evolution of lifespan in C. elegans. Nature 405, 296-297.
Wheeler, D.E., 1986. Developmental and physiological determinants of caste in social Hymenoptera - evolutionary implications. Am. Nat. 128, 13-34. Whitfield, C.W., Band, M.R., Bonaldo, M.F., Kumar, C.G., Liu, L., Pardinas, J.R., Robertson, H.M., Soares, M.B., Robinson, G.E., 2002. Annotated expressed sequence tags and cDNA microarrays for studies of brain and behavior in the honey bee. Genome Res. 12, 555-566. 